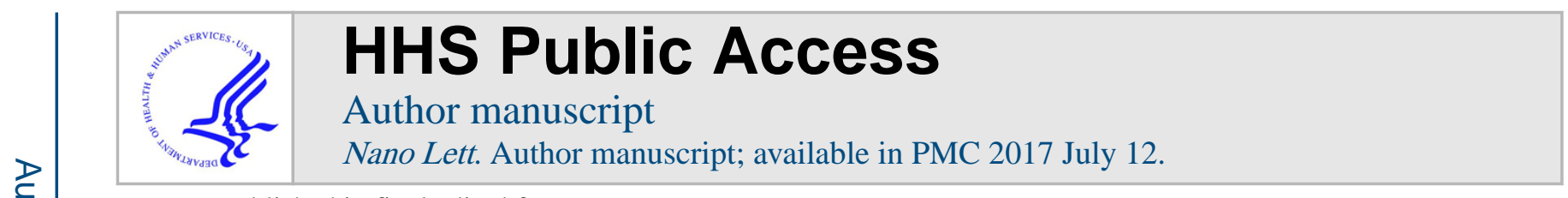

Published in final edited form as:

Nano Lett. 2015 November 11; 15(11): 7488-7496. doi:10.1021/acs.nanolett.5b03073.

\title{
Nanoparticle Mediated Tumor Vascular Disruption: A Novel Strategy in Radiation Therapy
}

\author{
Sijumon Kunjachan ${ }^{\star}, \dagger$, Alexandre Detappe ${ }^{\dagger, \ddagger}$, Rajiv Kumar ${ }^{\dagger, \S}$, Thomas Ireland ${ }^{\|}$, Lisa \\ Cameron $^{\perp}$, Douglas E. Biancur ${ }^{\dagger}$, Vincent Motto-Ros ${ }^{\ddagger}$, Lucie Sancey ${ }^{\ddagger}$, Srinivas Sridhar ${ }^{\dagger}$, , \\ G. Mike Makrigiorgos ${ }^{\dagger}$, and Ross I. Berbeco ${ }^{*}, \dagger$ \\ †Department of Radiation Oncology, Brigham and Women's Hospital, Dana-Farber Cancer \\ Institute and Harvard Medical School, Boston, Massachusetts 02115, United States \\ \#Institut Lumière Matière, Université Claude Bernard Lyon1-CNRS, Université de Lyon, 69007 \\ Lyon, France \\ §Nanomedicine Science and Technology Center and Department of Physics, Northeastern \\ University, Boston, Massachusetts 02115, United States \\ "LA-ICP-MS and ICP-ES Laboratories, Boston University, Boston, Massachusetts 02215, United \\ States \\ ${ }^{\perp}$ Department of Pediatric Oncology, Dana-Farber Cancer Institute, Boston, Massachusetts 02215, \\ United States
}

\begin{abstract}
More than $50 \%$ of all cancer patients receive radiation therapy. The clinical delivery of curative radiation dose is strictly restricted by the proximal healthy tissues. We propose a dual-targeting strategy using vessel-targeted-radio-sensitizing gold nanoparticles and conformal-image guided radiation therapy to specifically amplify damage in the tumor neoendothelium. The resulting tumor vascular disruption substantially improved the therapeutic outcome and subsidized the radiation/nanoparticle toxicity, extending its utility to intransigent or nonresectable tumors that barely respond to standard therapies.
\end{abstract}

\section{Graphical abstract}

\footnotetext{
*Corresponding Authors: Tel.: +1-214-250-0923. sijumon@gmail.com; skunjachan@lroc.harvard.edu. Tel.: +1-617-525-7136. rberbeco@lroc.harvard.edu.

The authors declare no competing financial interest.

Supporting Information

The Supporting Information is available free of charge on the ACS Publications website at DOI: 10.1021/acs.nanolett.5b03073.

Materials and methods to characterize different polymeric nanomedicines (PDF)
} 


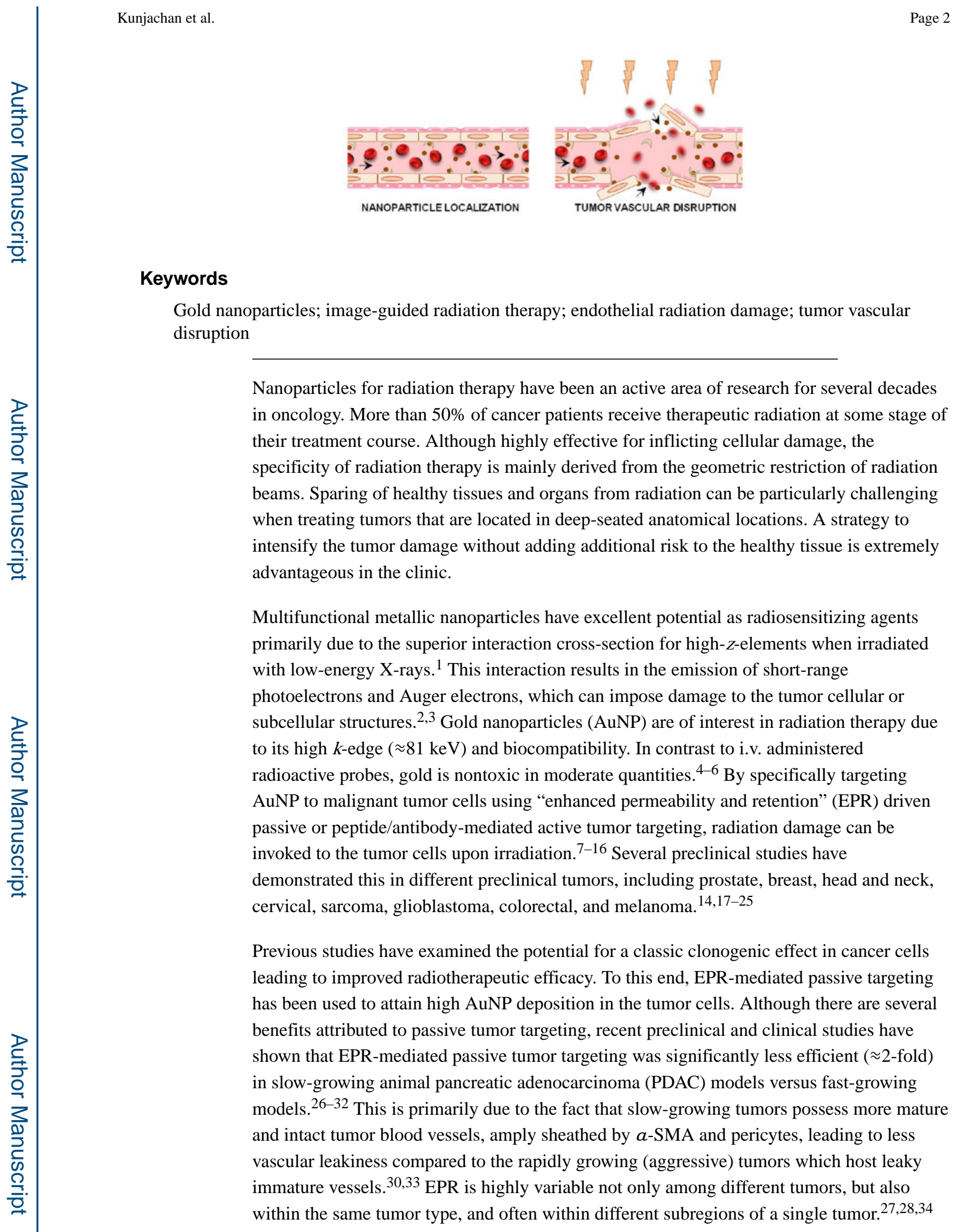

Nano Lett. Author manuscript; available in PMC 2017 July 12. 
Another critical factor that hampers drug/nanoparticle delivery to the tumor cells is the highly heterogeneous and dense fibrotic microenvironment of solid tumor (especially in PDAC tumor). It is therefore extremely difficult for anticancer drugs (for ex. Gemcitabine), proteins, peptides, or antibodies to diffuse and penetrate through the tumor interstitium to reach the cancer cells. ${ }^{29,35}$ In principle, this inherent tumor pathophysiology which regulates the poor diffusion of nanoparticles (beyond tumor vasculature and its periphery) is a serious limitation for cellular AuNP-mediated radiation therapy. ${ }^{35}$

Tumor neovasculature is an important target for both chemo- and radiation therapy. ${ }^{36-39}$ Studies show that even clonogenic cellular dysfunction due to radiation is primarily mediated by the microvascular endothelial damage. ${ }^{36,40-42}$ To this end, chemical vascular disrupting agents have been shown to be effective either alone or in combination with radiation therapy. However, recent clinical trials showed severe off-target toxicity issues associated with chemical vascular disrupting agent therapies. ${ }^{41-44}$ On the other hand, targeted-AuNP can minimize off-target localization and improve the overall accumulation at the tumor endothelium. In addition to this, the millimeter-scale accuracy of modern clinical image-guided radiation therapy can largely avoid AuNP activation in other healthy organs. This "two-fold targeting strategy" will minimize the normal tissue toxicity and consequently improve the therapeutic efficacy considerably. ${ }^{24,45-47}$

We have proposed a dual-targeting strategy by specific targeting of the tumor blood vasculature with targeted-gold nanoparticles and image-guided irradiation to improve radiation outcome by inducing vascular damage. Using Monte Carlo simulations, empirical electron range data, and analytical calculations, our previous studies clearly show that gold nanoparticles will contribute to substantial dose enhancement to the tumor endothelial cells even without a specific cellular uptake. ${ }^{45}$ In the following study, we experimentally validated the hypothesis that tumor-specific vascular disruption could be mediated by the administration of targeted gold nanoparticle followed by targeted irradiation in pancreatic tumor model. In our experimental design, gold nanoparticles were cofunctionalized with a targeting and imaging ligand and injected into mice-bearing (Panc-1) pancreatic tumor xenografts ( $\approx 1.2 \mathrm{mg} / \mathrm{g}$ of Au i.v.). The tumor was then irradiated (10 Gy), and the vesseldamage response was assessed using a series of different analytical/imaging techniques in vitro and in vivo. The schematic depiction illustrates some of the prototypical responses of vessel rupture postirradiation using gold nanoparticles, as further demonstrated in this study (Figure 1A-B).

PEGylated gold nanoparticles (AuNP) were prepared and cofunctionalized with Arg-GlyAsp (-RGD), a tumor neovascular targeting ligand, and a near-infrared dye, AF647. The bifunctional gold nanoparticle was prepared using a standard two-step process as described in the Materials and Methods section. Briefly, the THPC-stabilized nanoparticle were further functionalized to impart carboxylic $(-\mathrm{COOH})$ and amino $\left(-\mathrm{NH}_{2}\right)$ pendant groups which further undergoes EDC coupling reaction to covalently attach -RGD and AF-647 moieties, the targeting and the imaging agents respectively (Figure 2A). Besides the high $k$-edge, the relative ease of AuNP multifunctionalization, and its superior stability vis-a-vis for example, micelles, liposomes, lipid nanoparticles, antibody conjugates, etc., extends its utility in radiation therapy. PEG functionalization, by virtue of its higher hydrophilicity and stearic 
hindrance abilities, reduces the opsonization and improves the overall circulation kinetics and tumor accumulation. A near-infrared dye-AF647 was chemically tagged to the AuNP to facilitate fluorescence/confocal imaging. RGD (Arg-Gly-Asp), an oligopeptide, has high affinity for the transmembrane heterodimer $a_{\mathrm{v}} \beta_{3}$ integrin receptors which are highly overexpressed on the activated tumor neoendothelium. Since its inception in the 1980s, it has been used as a standard tumor vascular targeting ligand. ${ }^{28,48,49}$ For the remainder of this study, the neovascular-targeted (-RGD), PEGylated, and fluorophore-tagged AuNP formulation will be referred to as RGD:AuNP. With a spherical morphology, the core size of the RGD:AuNP was found to be $\approx 2-3 \mathrm{~nm}$ (by TEM imaging), and a hydrodynamic size of $\approx 8-10 \mathrm{~nm}$ was measured by DLS (Figure $2 \mathrm{~B}-\mathrm{C}$ ). Size plays a determinant role in predicting the radiotherapeutic benefits. Several studies have demonstrated that AuNP with the size of $\approx 10-12 \mathrm{~nm}$ can produce a high radiosensitization effect. ${ }^{50}$ The avg. zeta potential (surface charge) was $-11.07 \pm 1.07$ in PBS (7.4). Absorption and fluorescence spectra of RGD:AuNP showed the integrity of AF-647 postlabeling by listing distinct peaks at the anticipated absorption/fluorescence $\lambda_{\max }$ of 650/668 nm (Figure 2D).

For optimal therapeutic response, favorable in vivo accumulation and tumor blood vessel localization of nanoparticles in the tumor is crucial. To investigate the AuNP distribution profile in Panc-1- tumor bearing mice, we performed a series of high-resolution imaging techniques at early ( $1 \mathrm{~h}$, post-i.v.) and late ( $24 \mathrm{~h}$, post-i.v.) time points after RGD:AuNP administration. When STEM (scanning transmission electron microscopy) imaging and TEM (transmission electron microscopy) imaging assessed the early (1 h-p.i.) uptake kinetics of RGD-AuNP, LIBS (laser-induced breakdown spectroscopy), fluorescence/brightfield imaging, and ICPMS (inductively coupled plasma mass spectrometry) studies were used to trace the late localization (24 h-p.i.) of RGD-AuNP. Early distribution of RGD:AuNP in the tumor endothelium was detected as bright contrast signals in the STEMEDX imaging (Figure 3A-B). Distinct Au peaks simultaneously corroborating with the bright signals (inset) were obtained using the EDX spectral read-outs. Furthermore, in a functional tumor blood vessel, large clusters of nanoparticles were actively taken up by the tumor endothelial cells as observed using TEM imaging (Figure 3C-E). In line with the previous reports, the uptake of RGD:AuNP may be primarily mediated by the active clathrin/caveolae- mediated endocytosis. ${ }^{51-54}$ However, using non targeted gold nanoparticles, no active endothelial uptake was observed (see Supporting Information Figure S1). At 24 h post-i.v. injection, localization of RGD:AuNP was visualized close to the tumor blood vessels (Figure 3F-H). LIBS imaging, a novel advanced technique to specifically detect heavy metals/elements showed heterogeneous distribution of gold (RGD:AuNP) in the tumor tissue. ${ }^{55,56}$ The spectral read-outs resonated with corresponding peaks for gold in the treated samples vs nontreated samples (Figure 3I-L). More to it, the (bio) distribution of RGD:AuNP in the tumor and other vital organs were measured with IC-PMS at 24 h-posti.v. Preferentially high accumulation in the tumor was apparently observed (Figure 3M). Other reported studies have shown that $\approx 10 \mathrm{~nm}$-sized gold nanoparticles that accumulate in the liver (kupffer cells) are eventually cleared by the hepatobiliary pathways. ${ }^{57}$ In addition to this, the collimated radiation setup that we employed largely reduces RGD:AuNP activation in off-target organs such as the liver. This has been confirmed by assessing the radiation dose distribution in tumor and other organs (which is explained in the upcoming sections). 
Due to the presence of overexpressed integrin receptors in the activated tumor endothelium of PDAC tumors, strong colocalization between RGD:AuNP and tumor blood vessels was observed in vivo at 24 h-p.i. using fluorescence imaging (Figure $3 \mathrm{~N}$ ).

In the following in vitro and in vivo results, the group receiving both RGD:AuNP and irradiation is referred to as +RGD:AuNP/+IR, the group receiving RGD:AuNP alone is referred to as +RGD:AuNP/-IR, the group receiving irradiation alone is referred to as -RGD:AuNP/+IR, and the group receiving neither AuNP nor irradiation is -RGD:AuNP/ -IR. To validate the hypothesis of endothelial damage using RGD:AuNP upon irradiation, we performed an in vitro study using human umbilical vein endothelial cells (HUVEC) overexpressing the $a_{\mathrm{v}} \beta_{3}$ integrins during proliferation. By using a set of treated and nontreated controls (+RGD:AuNP/+IR, +RGD:AuNP/-IR, -RGD:AuNP/+IR, and -RGD:AuNP/-IR), three different radiation doses (10, 5, and $0 \mathrm{~Gy}$ ) were tested in vitro. Results obtained from the crystal violet assay displayed obvious qualitative differences between the treated (10 Gy, $5 \mathrm{~Gy}$ ) and the nontreated ( $0 \mathrm{~Gy}$ ) samples (Figure 4A). Unlike colony forming tumor cells, endothelial cells formed a single monolayer in the plates. The cells were therefore lysed to extract the stain from viable cells and the absorbance was measured at $590 \mathrm{~nm}$. A statistically significant difference $(P<0.05)$ in the HUVEC survival was observed for the + RGD:AuNP/+IR (10 Gy) sampled vs the unirradiated controls (58\% vs 98\%) (Figure 4B). In addition, the sensitivity enhancement ratios (SER, defined as the ratio of survival fractions after irradiation with and without nanoparticles) were calculated at 10 and $5 \mathrm{~Gy}$, and was found to be $1.2 \pm 0.022$ and $1.0 \pm 0.028$, respectively. Furthermore, on assessing the morphological changes in the HUVEC cells post-IR using phase contrast microscopy, increased endothelial cell rupture was observed for the radiation-treated ones (+RGD:AuNP/+IR) compared to nontreated samples (+RGD:AuNP/-IR and -RGD:AuNP/ -IR) samples (Figure 4C), demonstrating the effect of the radiation. Nearly 2 -fold differences (33\% vs $41 \%$ vs $95 \%$ ) in the cell survival were observed between the RGD:AuNP treated/irradiated group vs nontreated group (Figure 4D). Furthermore, the uptake of nanoparticles (RGD-AuNP) was also compared with the respective survival in these in vitro endothelial models (see supplementary Figure S2).

Clinical radiation therapy of pancreatic cancer is often limited by the close proximity of organs-at-risk such as liver, duodenum, spleen, and kidneys. In our study, we used a Small Animal Radiation Research Platform (SARRP) to perform image-guided radiation studies (Xtrahl, Inc.). By applying collimated radiation beams from two orthogonal angles, optimal tumor coverage was achieved, and the exposure to other organs was grossly minimized (Figure 5A). To corroborate this, we performed image-guided dosimetry studies (Muriplan V.1.3.0) to determine the dose distribution in the tumor and normal tissues (Figure 5B). The results clearly indicate that $>80 \%$ of the tumor region received a radiation dose of at least 10 Gy, while the normal tissues were largely spared $(<5 \%)$ (Figure 5C-D). Further histological staining of the adjacent muscle tissue showed no apparent radiation damage (see supplementary Figure S3). This setup ensures maximum dose at the tumors with minimal effect on other healthy tissues or organs.

We further evaluated the potential of RGD:AuNP to induce tumor vascular damage in Panc-1 tumor xenografts at $24 \mathrm{~h}$ post-IR. 3D-confocal imaging was performed on excised 
tumor tissue preinjected with a standard vessel marker, FITC-dextran (70 kDa; $60 \mu \mathrm{L}$ of 1 $\mathrm{mg} / \mathrm{mL}$ ). For the +RGD:AuNP/+IR cohort, a high degree of specific vascular damage was observed compared to the respective controls (Figure 6A-D). The tumor endothelial cells were severely damaged by the +RGD:AuNP/+IR combined treatment. However, in the respective controls, intact blood vessels with consistent endothelial integrity/functionality and uniformity were noticed (Figure 6B-D). Of note, two different types of vascular damage were apparently visible: fragmented vessels and diffused vessels (see supplementary Figure S4). Bright-field imaging was further employed to assess the morphological damage at the single vessel scale. Complete rupture of tumor vascular endothelium and damage to the vessel was clearly evident further confirming the previous results (Figure 6A). Partially segregated endothelial cells were present in the vicinity of damage site (cf. arrowheads). Essentially, the control batch showed normal tumor vascularity and endothelial integrity without any rupture (Figure $6 \mathrm{~F}-\mathrm{H}$ ). We performed CD34 stainings to specifically detect the damage at the tumor microvasculature. To this end, large depletion of vessel structure and integrity was seen in the +RGD:AuNP/+IR samples compared to the respective controls (Figure 6I-L). At higher magnifications (2500×), TEM imaging clearly showed unambiguous damage to the tumor endothelium and the disruption of tumor blood vessel (Figure 6M). In the controls, however, endothelial cells were intact and sufficiently protected by the basement membrane (BM) and pericytes (Figure $6 \mathrm{~N}-\mathrm{P}$ ). All of these observations using diverse techniques to evaluate different aspects of damage clearly show that the targeted nanoparticles induced specific, catastrophic vascular damage in pancreatic tumors following irradiation.

In order to assess the direct radiation damage response at the molecular level, we measured overall tumor suppression and specific DNA damage (double-strand breaks). Considerable reduction in the tumor proliferation and massive cell death was observed for the + RGD:AuNP/+IR samples compared to the respective controls using H\&E staining (Figure 7A-D). Assuming the effect to be a more generic tumor suppression response, we further investigated the possibilities for specific radiation damage. The effect of irradiation on the tumor and tumor endothelial cells at the DNA level was measured by using $\gamma-\mathrm{H} 2 \mathrm{AX}$ staining, taking into account recent experimental evidence of the temporal variations of $\gamma$ $\mathrm{H} 2 \mathrm{AX}$ positive foci formation in tumor tissue. ${ }^{58}$ We irradiated tumors \pm RGD:AuNP and dissected the tumors 30-40 min post-IR. $\gamma$-H2AX foci-formation in the tumor specimens showed a high degree of DNA double-strand breaks in the +RGD:AuNP/+IR samples (Figure 7E) compared to the controls (Figure 7F-H). Additionally, a large effect on the tumor endothelial cells was also observed (Figure 7I-J). Quantification of the DNA double strand breaks by $\gamma \mathrm{H} 2 \mathrm{AX}$ staining showed $\approx 3$-fold increase $(P<0.001)$ in the radiation specific DNA damage in the "nanoparticle-radiation" group (+RGD:AuNP/+IR: 57\%) compared to the "radiation only" group (-RGD:AuNP/+IR: 19\%) and almost $\approx 10$-fold difference $(P<0.001)$ compared to other controls (+RGD:AuNP/-IR: 6\% and -RGD:AuNP/-IR: 6\%) (Figure 7K).

Our experimental findings support the original concept of targeted gold nanoparticles to induce specific tumor vascular damage during radiation therapy. Unlike cellular targeting, which is often severely restricted by tissue (and physical) barriers, activated tumor endothelial targeting enables direct systemic access of nanoparticles to the overexpressed 
vascular targeting motifs. Moreover, shutting off a tumor blood capillary can affect numerous proliferating cancer cells, and an antitumorigenic (or antiangiogenic) effect can be indirectly potentiated. By means of inducing both direct and indirect tumor cell killing mechanisms, we anticipate that this innovative treatment modality has promising clinical potential. Clinically administered chemical vascular disrupting agents have suffered from serious toxicity concerns. Combrestatin, a clinical vascular disrupting agent, showed fatal dose-limiting side effects including pulmonary embolism and coronary vasospasm when tested in human trials. In our approach, however, the activating radiation beams can be exclusively restricted to the tumor (containing actively targeted gold nanoparticles). This dual-targeting platform could help maximize the therapeutic index by (1) increasing tumor damage and/or (2) reducing the amount of radiation dose needed to provide the same therapy effect and therefore limiting collateral damage to healthy tissues. The gold nanoparticles which are localized at other parts of the body (largely unaffected by radiation) will eventually be cleared by several phase degradation/detoxification mechanisms. ${ }^{59}$ The further impact of nanoparticle mediated vascular damage using radiation therapy in terms of halting tumor blood vessel functionality and its downstream effects are currently under investigation. Therapy-induced hypoxia may be a challenge to a fractionated clinical approach, but this needs to be independently investigated. To summarize, this dual-targeting strategy holds great translational potential in radiation oncology. Application of this concept to other intransigent or nonresectable tumor types for which radiation delivery is limited by adjacent organs adds to the potential clinical impact. The data presented in this paper represent the first in-depth experimental investigation of tumor vascular disruption with metallic nanoparticles, a novel strategy in radiation therapy.

\section{Supplementary Material}

Refer to Web version on PubMed Central for supplementary material.

\section{Acknowledgments}

We acknowledge the efforts by Dr. Houari Korideck for assistance with the SARRP irradiations at Dana-Farber Cancer Institute, and the histology core facility at Brigham and Women's Hospital and Harvard Medical School, and the TEM imaging core facility at Harvard Medical School. This project was supported, in part, by a grant from the JCRT Foundation and by award numbers R03 CA164645 and R21 CA188833 from the National Cancer Institute (NCI). The content of this manuscript is solely the responsibility of the authors and does not necessarily represent the official views of the NCI or NIH.

\section{References}

1. Hainfeld JF, Dilmanian FA, Slatkin DN, Smilowitz HM. J Pharm Pharmacol. 2008; 60:977-985. [PubMed: 18644191]

2. Jelveh S, Chithrani DB. Cancers. 2011; 3:1081-1110. [PubMed: 24212654]

3. Kumar R, Korideck H, Ngwa W, Berbeco RI, Makrigiorgos GM, Sridhar S. Transl Cancer Res. 2013; 72:2.

4. Almeida JP, Chen AL, Foster A, Drezek R. Nanomedicine (London, U K). 2011; 6:815-835.

5. Thakor AS, Jokerst J, Zavaleta C, Massoud TF, Gambhir SS. Nano Lett. 2011; 11:4029-36. [PubMed: 21846107]

6. Thakor AS, Luong R, Paulmurugan R, Lin FI, Kempen P, Zavaleta C, Chu P, Massoud TF, Sinclair R, Gambhir SS. Sci Transl Med. 2011; 3:79ra33. 
7. Yang YS, Carney RP, Stellacci F, Irvine DJ. ACS Nano. 2014; 8:8992-9002. [PubMed: 25123510]

8. Chithrani DB, Dunne M, Stewart J, Allen C, Jaffray DA. Nanomedicine. 2010; 6:161-169. [PubMed: 19447206]

9. Chithrani BD, Ghazani AA, Chan WC. Nano Lett. 2006; 6:662-668. [PubMed: 16608261]

10. Berbeco RI, Korideck H, Ngwa W, Kumar R, Patel J, Sridhar S, Johnson S, Price BD, Kimmelman A, Makrigiorgos GM. Radiat Res. 2012; 178:604-608. [PubMed: 23148509]

11. Jain S, Coulter JA, Butterworth KT, Hounsell AR, McMahon SJ, Hyland WB, Muir MF, Dickson GR, Prise KM, Currell FJ, Hirst DG, O’Sullivan JM. Radiother Oncol. 2014; 110:342-347. [PubMed: 24444528]

12. Hainfeld JF, Smilowitz HM, O’Connor MJ, Dilmanian FA, Slatkin DN. Nanomedicine (London, U K). 2013; 8:1601-1609.

13. Dorsey JF, Sun L, Joh DY, Witztum A, Kao GD, Alonso-Basanta M, Avery S, Hahn SM, Al ZA, Tsourkas A. Transl Cancer Res. 2013; 2:280-291. [PubMed: 25429358]

14. Joh DY, Sun L, Stangl M, Al Zaki ZA, Murty S, Santoiemma PP, Davis JJ, Baumann BC, AlonsoBasanta M, Bhang D, Kao GD, Tsourkas A, Dorsey JF. PLoS One. 2013; 8:62425.

15. Ngwa W, Kumar R, Sridhar S, Korideck H, Zygmanski P, Cormack RA, Berbeco R, Makrigiorgos GM. Nanomedicine (London, U K). 2014; 9:1063-1082.

16. Chen W, Ayala-Orozco C, Biswal NC, Perez-Torres C, Bartels M, Bardhan R, Stinnet G, Liu XD, Ji B, Deorukhkar A, Brown LV, Guha S, Pautler RG, Krishnan S, Halas NJ, Joshi A. Nanomedicine (London, U K). 2014; 9:1209-1222.

17. Tree AC, Ostler P, Hoskin P, Dankulchai P, Nariyangadu P, Hughes RJ, Wells E, Taylor H, Khoo VS, van As NJ. Clin Oncol (R Coll Radiol). 2014; 26:757-761. [PubMed: 25193299]

18. Atkinson RL, Zhang M, Diagaradjane P, Peddibhotla S, Contreras A, Hilsenbeck SG, Woodward WA, Krishnan S, Chang JC, Rosen JM. Sci Transl Med. 2010; 2:55ra79.

19. Lee J, Chatterjee DK, Lee MH, Krishnan S. Cancer Lett. 2014; 347:46-53. [PubMed: 24556077]

20. Masood R, Roy I, Zu S, Hochstim C, Yong KT, Law WC, Ding H, Sinha UK, Prasad PN. Integr Biol (Camb). 2012; 4:132-141. [PubMed: 22159374]

21. Hainfeld JF, Dilmanian FA, Zhong Z, Slatkin DN, Kalef-Ezra JA, Smilowitz HM. Phys Med Biol. 2010; 55:3045-59. [PubMed: 20463371]

22. Zhang XD, Chen J, Luo Z, Wu D, Shen X, Song SS, Sun YM, Liu PX, Zhao J, Huo S, Fan S, Fan F, Liang XJ, Xie J. Adv Healthcare Mater. 2014; 3:133-141.

23. Joh DY, Kao GD, Murty S, Stangl M, Sun L, Al Zaki ZA, Xu X, Hahn SM, Tsourkas A, Dorsey JF. Transl Oncol. 2013; 6:722-731. [PubMed: 24466375]

24. Diagaradjane P, Shetty A, Wang JC, Elliott AM, Schwartz J, Shentu S, Park HC, Deorukhkar A, Stafford RJ, Cho SH, Tunnell JW, Hazle JD, Krishnan S. Nano Lett. 2008; 8:1492-1500. [PubMed: 18412402]

25. Chang MY, Shiau AL, Chen YH, Chang CJ, Chen HH, Wu CL. Cancer Sci. 2008; 99:1479-1484. [PubMed: 18410403]

26. Matsumura Y, Oda T, Maeda H. Gan To Kagaku Ryoho. 1987; 14:821-829. [PubMed: 2952066]

27. Maeda H, Wu J, Sawa T, Matsumura Y, Hori KJ. Controlled Release. 2000; 65:271-284.

28. Kunjachan S, Pola R, Gremse F, Theek B, Ehling J, Moeckel D, Hermanns-Sachweh B, Pechar M, Ulbrich K, Hennink WE, Storm G, Lederle W, Kiessling F, Lammers T. Nano Lett. 2014; 14:972981. [PubMed: 24422585]

29. Cabral H, Matsumoto Y, Mizuno K, Chen Q, Murakami M, Kimura M, Terada Y, Kano MR, Miyazono K, Uesaka M, Nishiyama N, Kataoka K. Nat Nanotechnol. 2011; 6:815-823. [PubMed: 22020122]

30. Harrington KJ, Mohammadtaghi S, Uster PS, Glass D, Peters AM, Vile RG, Stewart JS. Clin Cancer Res. 2001; 7:243-254. [PubMed: 11234875]

31. Kunjachan S, Ehling J, Storm G, Kiessling F, Lammers T. Chem Rev. 2015; doi: 10.1021/ cr500314d

32. Kunjachan S, Jayapaul J, Mertens ME, Storm G, Kiessling F, Lammers T. Curr Pharm Biotechnol. 2012; 13:609-622. [PubMed: 22214503]

33. Siegel RL, Miller KD, Jemal A. Ca-Cancer J Clin. 2015; 65:5-29. [PubMed: 25559415] 
34. Maeda H, Matsumura Y. Adv Drug Delivery Rev. 2011; 63:129-130.

35. Hauert S, Berman S, Nagpal R, Bhatia SN. Nano Today. 2013; 8:566-576. [PubMed: 25009578]

36. Garcia-Barros M, Paris F, Cordon-Cardo C, Lyden D, Rafii S, Haimovitz-Friedman A, Fuks Z, Kolesnick R. Science. 2003; 300:1155-1159. [PubMed: 12750523]

37. Arap W, Pasqualini R, Ruoslahti E. Science. 1998; 279:377-380. [PubMed: 9430587]

38. Cooney MM, van Heeckeren HW, Bhakta S, Ortiz J, Remick SC. Nat Clin Pract Oncol. 2006; 3:682-692. [PubMed: 17139319]

39. Banerjee D, Harfouche R, Sengupta S. Vasc Cell. 2011; 3:3. [PubMed: 21349160]

40. Potten CS. Int J Radiat Biol. 1990; 58:925-973. [PubMed: 1978853]

41. Siemann DW, Rojiani AM. Int J Radiat Oncol, Biol, Phys. 2005; 62:846-853. [PubMed: 15936569]

42. Siemann DW, Horsman MR. Cell Tissue Res. 2009; 335:241-248. [PubMed: 18752004]

43. Mauceri HJ, Hanna NN, Wayne JD, Hallahan DE, Hellman S, Weichselbaum RR. Cancer Res. 1996; 56:4311-4314. [PubMed: 8813113]

44. Hinnen P, Eskens FA. Br J Cancer. 2007; 96:1159-1165. [PubMed: 17375046]

45. Berbeco RI, Ngwa W, Makrigiorgos GM. Int J Radiat Oncol, Biol, Phys. 2011; 81:270-276. [PubMed: 21163591]

46. Berbeco RI, Korideck H, Ngwa W, Kumar R, Patel J, Sridhar S, Johnson S, Price BD, Kimmelman A, Makrigiorgos GM. Radiat Res. 2012; 178:604-608. [PubMed: 23148509]

47. Rahman WN, Bishara N, Ackerly T, He CF, Jackson P, Wong C, Davidson R, Geso M. Nanomedicine. 2009; 5:136-142. [PubMed: 19480049]

48. Ruoslahti E, Pierschbacher MD. Science. 1987; 238:491-497. [PubMed: 2821619]

49. Ruoslahti E. Matrix Biol. 2003; 22:459-65. [PubMed: 14667838]

50. Zhang XD, Wu D, Shen X, Chen J, Sun YM, Liu PX, Liang XJ. Biomaterials. 2012; 33:64086419. [PubMed: 22681980]

51. Chithrani BD, Chan WC. Nano Lett. 2007; 7:1542-1550. [PubMed: 17465586]

52. Jiang W, Kim BY, Rutka JT, Chan WC. Nat Nanotechnol. 2008; 3:145-150. [PubMed: 18654486]

53. Chithrani BD, Stewart J, Allen C, Jaffray DA. Nanomedicine. 2009; 5:118-127. [PubMed: 19480047]

54. Yang C, Uertz J, Yohan D, Chithrani BD. Nanoscale. 2014; 6:12026-12033. [PubMed: 25182693]

55. Sancey L, Motto-Ros V, Busser B, Kotb S, Benoit JM, Piednoir A, Lux F, Tillement O, Panczer G, Yu J. Sci Rep. 2014; 4:6065. [PubMed: 25338518]

56. Sancey L, Kotb S, Truillet C, Appaix F, Marais A, Thomas E, van der Sanden B, Klein JP, Laurent B, Cottier M, Antoine R, Dugourd P, Panczer G, Lux F, Perriat P, Motto-Ros V, Tillement O. ACS Nano. 2015; 9:2477-2488. [PubMed: 25703068]

57. Hirn S, Semmler-Behnke M, Schleh C, Wenk A, Lipka J, Schaffler M, Takenaka S, Moller W, Schmid G, Simon U, Kreyling WG. Eur J Pharm Biopharm. 2011; 77:407-416. [PubMed: 21195759]

58. Herter-Sprie GS, Korideck H, Christensen CL, Herter JM, Rhee K, Berbeco RI, Bennett DG, Akbay EA, Kozono D, Mak RH, Makrigiorgos MG, Kimmelman AC, Wong KK. Nat Commun. 2014; 5:5870. [PubMed: 25519892]

59. Kolosnjaj-Tabi J, Javed Y, Lartigue L, Volatron J, Elgrabli D, Marangon I, Pugliese G, Caron B, Figuerola A, Luciani N, Pellegrino T, Alloyeau D, Gazeau F. ACS Nano. 2015; 9:7925. [PubMed: 26168364] 


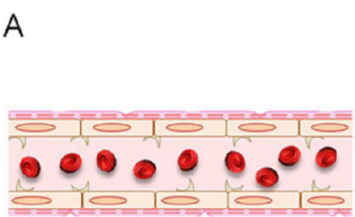

TUMOR BLOOD VESSEL

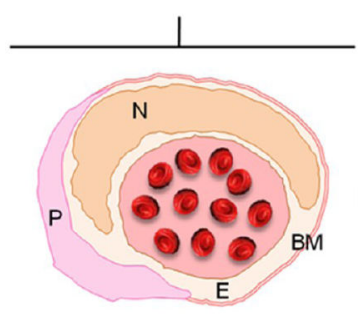

B

PDAC tumor RGD:AuNP (5-8 $\left.\mathrm{mm}^{2}\right)$

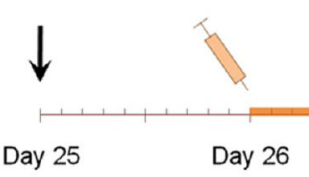

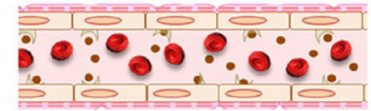

NANOPARTICLE LOCALIZATION

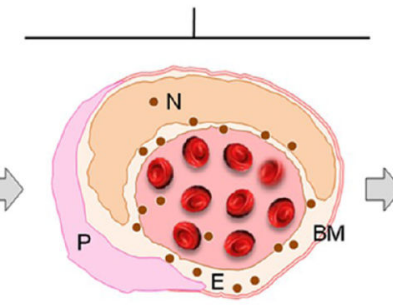

Tumor irradiation

FITC-DEX injection
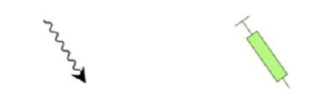

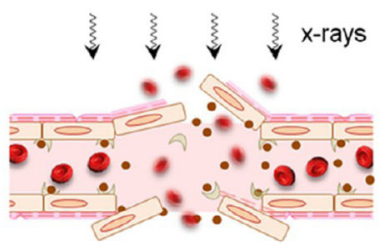

TUMOR VASCULAR DISRUPTION

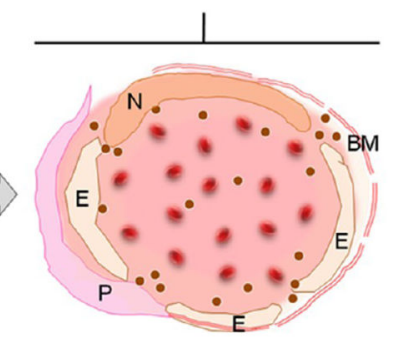

Confocal imaging / IHC Validation of 'disruption'

Day 28

Day 27



Figure 1.

Experimental design and concept. (A) Schematic illustration of a tumor angiogenic blood vessel which, after active (vascular) targeting by gold nanoparticles to the $a_{\mathrm{v}} \beta_{3}$ integrin receptors and subsequent irradiation, suffers tumor endothelial disruption. The crosssectional view depicts some of the prototypical responses related to "vascular disruption" where the endothelium (E), pericytes (P), basement membrane (BM), and endothelial nuclei (N) undergo morphological changes and membrane destabilization leading to vessel rupture. (B) Roughly 25 days after s.c. tumor inoculation in $\mathrm{NCr}$ nude mice, $\approx 5-8 \mathrm{~mm}^{2}$ sized Panc-1 tumor xenografts were obtained. The gold nanoparticles (referred to as RGD:AuNP) were synthesized and functionalized with the targeting ligand (RGD) and the imaging agent (AF-647). After proper characterization, RGD:AuNP (1.25 mg/mL equiv. Au in $200 \mu \mathrm{L}$ ) was administered into tumor-bearing mice and irradiated at $24 \mathrm{~h}$ post-i.v. injection. FITCdextran $(70 \mathrm{kDa} ; 1 \mathrm{mg} / \mathrm{mL})$ was injected at $24 \mathrm{~h}$ after the irradiation, and the mouse was sacrificed in 5-10 min to excise the tumor and other vital organs for further investigations. 

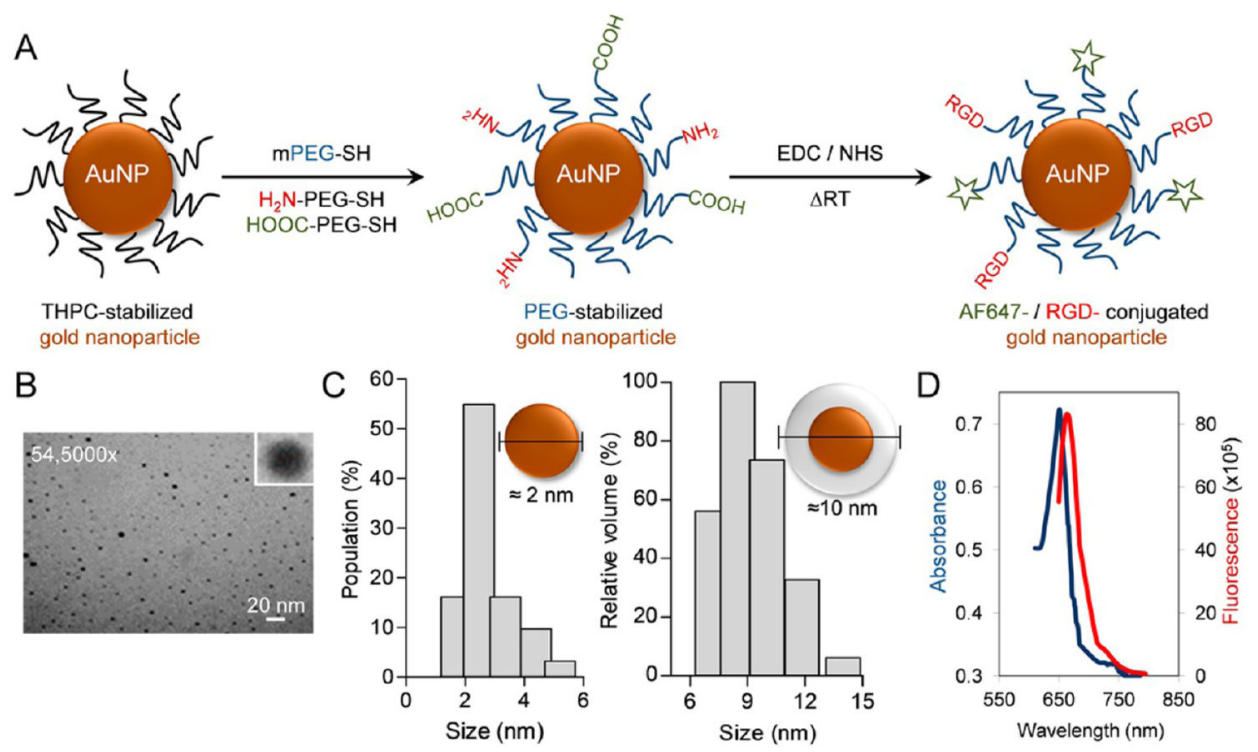

Figure 2.

Chemical synthesis and characterization of functionalized gold nanoparticles for vascular tumor targeting. (A) Schematic representation of stepwise synthesis where PEGylated gold nanoparticles (AuNP) were bifunctionalized with Arg-Gly-Asp (RGD) and a near-infrared imaging agent (AF647). The resultant product, PEG-RGD-AuNP-AF647 (or RGD:AuNP), was further purified and characterized. (B) RGD:AuNP showed spherical surface morphology (cf. inset) when analyzed using TEM imaging. (C) The particle size (core and hydrodynamic size) was measured by both TEM and DLS, and its core size was estimated to be $\approx 2-3 \mathrm{~nm}$, whereas the hydrodynamic size was $\approx 8-10 \mathrm{~nm}$. (D) The absorption and fluorescence spectra of RGD:AuNP was recorded postlabeling, and it was found to be $\lambda_{\max }$ of 650/668 nm, in agreement with previous reported studies. 


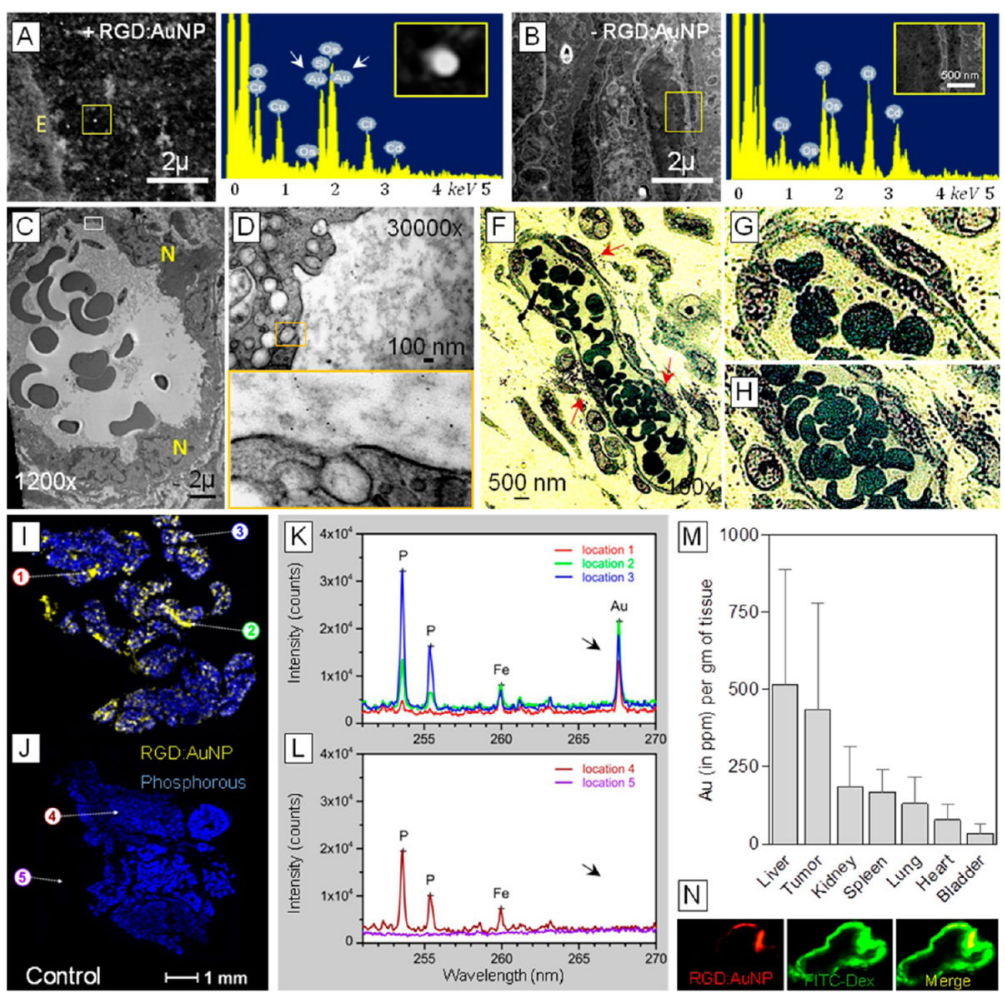

Figure 3.

RGD:AuNP localization in vivo and tumor vascular targeting. (A-B) STEM (scanning transmission electron microscopy) imaging detects the presence of targeted nanoparticles (indicated by bright contrast) on the tumor vessels at $1 \mathrm{~h}$ after RGD-AuNP injection in Panc-1 tumor bearing mice. The corresponding EDX spectral read-outs show distinct peaks which are specific for $\mathrm{Au}$ (see insets) in the samples. Apparently no gold was seen in the respective controls. (C-E) TEM images show the early uptake $(1 \mathrm{~h})$ of nanoparticles by the tumor endothelial cells in vivo. Higher magnified images (manual) indicate clathrin/ caveolae-mediated uptake at early/late endosomal stages. E: endothelium; N: nucleus. (F-H) Bright field images show the RGD:AuNP localization close to the vessels at $1 \mathrm{~h}$ postadministration. Higher manual magnification of those images show the formation of AuNP aggregates close to the tumor endothelium. (I-L) Laser-induced breakdown spectroscopy (LIBS) imaging was carried out to specifically confirm the presence of RGD:AuNP localization within the tumor 24 h post-i.v. Unlike TEM imaging, LIBS facilitates real-time monitoring of Au distribution within the tumors and directly correlates with the respective wavelengths in the corresponding spectral read-outs. (M) Biodistribution of RGD:AuNP in other organs was measured 24 h post-i.v. by ICPMS $(n=3)$, and the amount of Au was quantified based on the corresponding organ weight. The values represent average \pm SD. (N) The colocalization of RGD:AuNP with FITC-dextran-labeled tumor endothelium was analyzed using fluorescence imaging. Strong colocalization is observed with (AF647-coupled) nanoparticle near the endothelial cells. 
A

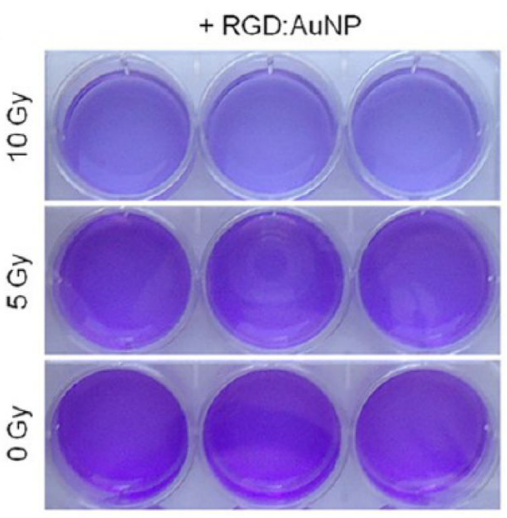

B

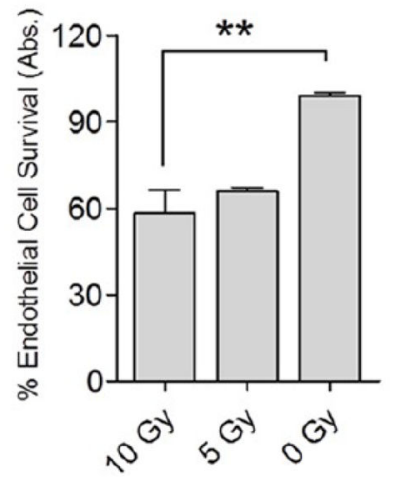

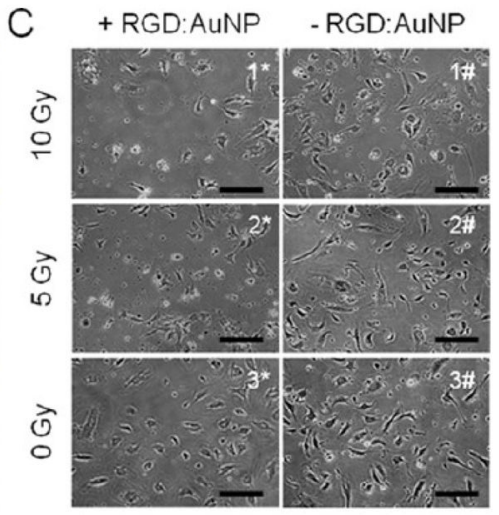

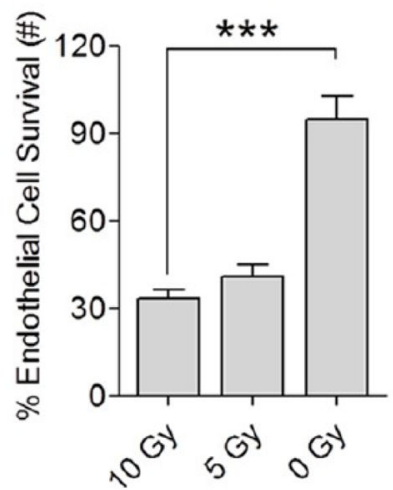

Figure 4.

In vitro radiation enhancement study. Human umbilical vein endothelial cells were treated $( \pm)$ RGD:AuNP (indicated by * and \#, respectively) and exposed to three different radiation doses: 0,5 , and $10 \mathrm{~Gy}$. All four treatment conditions were replicated in this study: +RGD:AuNP/+IR, +RGD:AuNP/-IR, -RGD:AuNP/+IR, and -RGD:AuNP/-IR. (A-B) Crystal violet assay was performed on endothelial cells 1-week postirradiation to detect the differences in the cell survival. The cells were lysed to extract and quantify crystal violet, by measuring its absorbance using a spectrophotometer at $590 \mathrm{~nm}$. The values represent mean \pm $\mathrm{SD}$, and all of the data was normalized to its respective nontreated controls. $* * P<0.0025$. (C-D) Phase contrast microscopy was performed 1-week post-IR to visualize the proliferation (or survival) of the endothelial cells. Apparently, clear differences in the cell density was observed between the treated vs nontreated group. Bar: 10x. Further quantification of the phase contrast microscopic images was carried out by counting the viable cells/frame (using ImageJ) in RGD:AuNP treated vs nontreated in both irradiated and nonirradiated controls. In total, $n=30$ representative images/condition were analyzed. The values represent the mean $\pm \mathrm{SD}$, and all of the data was normalized to its respective nontreated controls. $* * * P<0.0001$. 

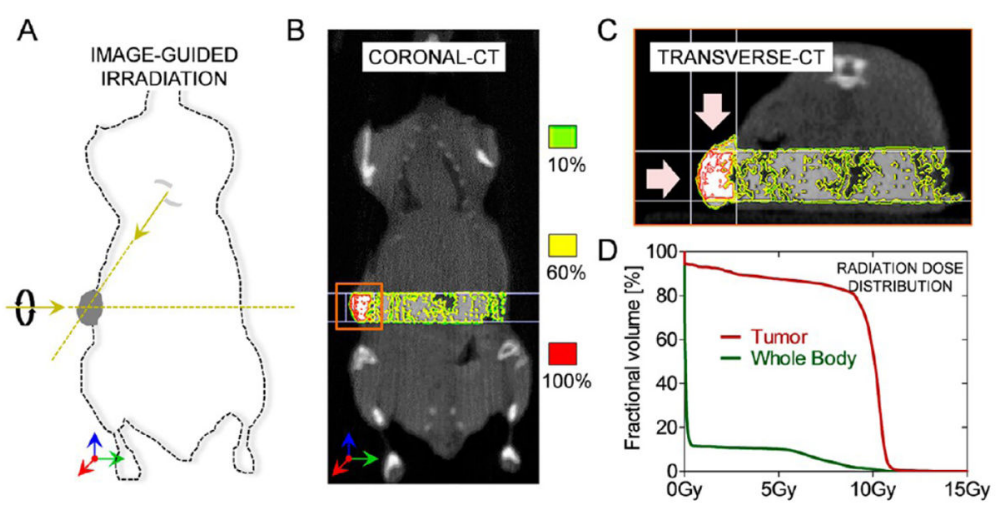

Figure 5.

Small animal radiation research platform and radiation dose distribution in vivo. (A) Radiation setup where each Panc-1 tumor xenograft was irradiated with 10 Gy (220 kVp) radiation beam. We used orthogonal, collimated beams in order to maximize the radiation dose exposure to the tumors and minimize the effect on (off-target) healthy tissues. (B) The representative coronal $\mathrm{CT}$ image shows the real-time distribution of radiation dose in the tumor and the surrounding tissue for RGD:AuNP-treated mouse with Panc-1 tumors. The isodose distribution shows high dose (95\%-100\%) in the tumor compared to the surrounding tissue. (C) Transverse (axial) section shows the isodose distribution specifically in the tumor. (D) The quantification of radiation doses from the cone beam CT images shows that more than $80 \%$ of the tumor received 10 Gy compared to the whole body. 


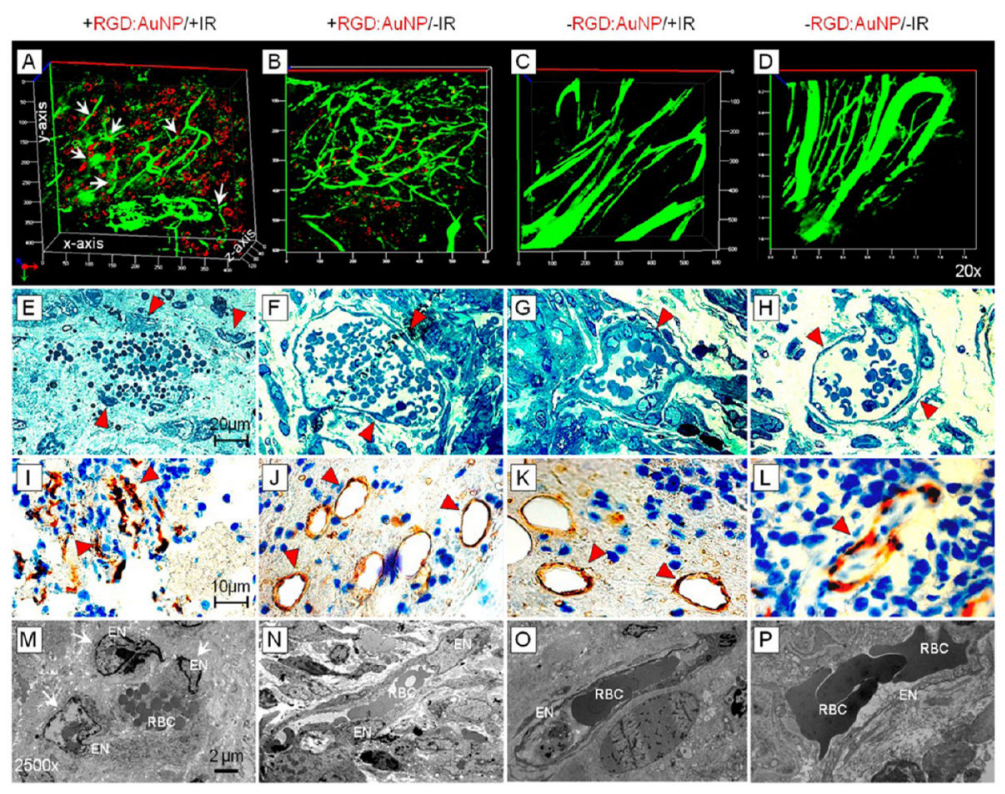

Figure 6.

Imaging tumor vascular disruption. (A-D) Confocal imaging with RGD:AuNP (red) and FITC-Dextran (green) shows a high degree of vascular damage at the indicated locations (white arrows) and the presence of highly dense RGD:AuNPs in its close proximity, compared to the respective controls. $(\mathrm{E}-\mathrm{H})$ Bright field imaging shows damaged endothelial cells, and a change in the morphology of red blood cells clearly demonstrates loss of functionality for some of these vessels. The control samples showed intact vessels and prominent endothelium. (I-L) CD34 IHC shows collapsed vessels and altered morphology (red marker) compared to the respective controls. (M-P) TEM imaging clearly confirms the endothelial rupture (see arrows). In the +RGD:AuNP/+IR samples, endothelial cells were detached and the cell nuclei damaged. The control samples showed high integrity and intact morphology. EN: Endothelial nucleus; RBC: red blood cells; BM: basement membrane. 


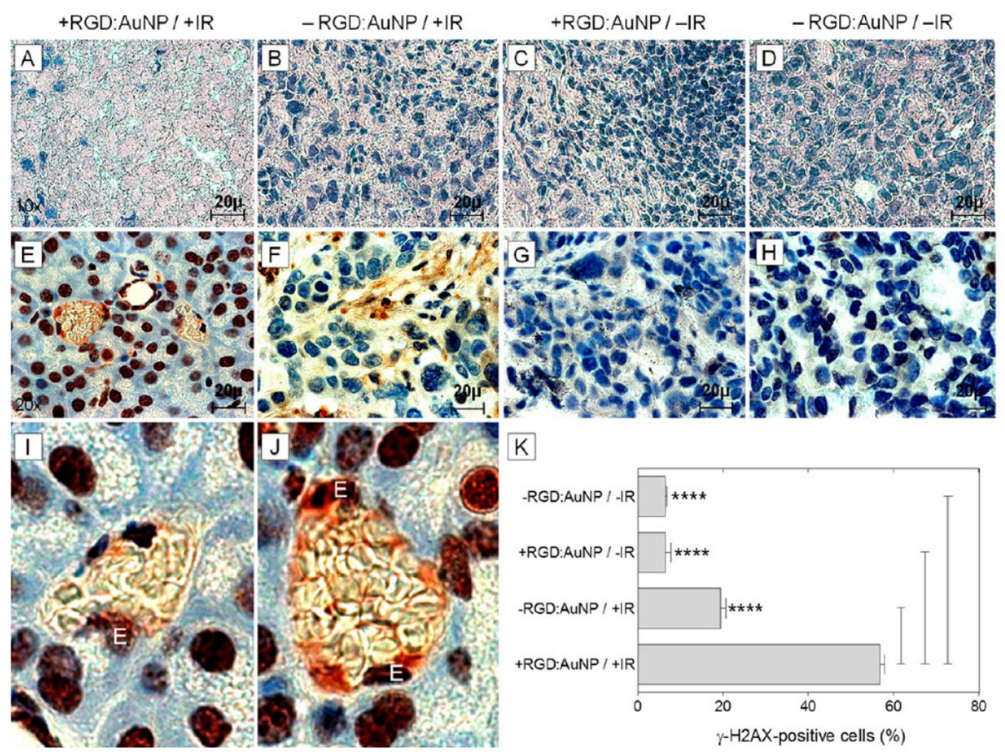

Figure 7.

Assessing radiation outcome and specific DNA damage. (A-D) The H\&E staining revealed the effect of radiation on the Panc-1 tumor xenograft. Massive cell death was observed in the RGD:AuNP treated samples compared to respective controls. (E-H) By $\gamma-\mathrm{H} 2 \mathrm{AX}$ staining, we measured radiation induced DNA double strand breaks in the tumor. Color: dark-brown: $\gamma$-H2AX-positive nuclei; blue: Hematoxylin-positive nuclei. (I-J) This effect was remarkable in the tumor blood vessels especially in the endothelial cell nucleus (denoted by "E") showing high degree of radiation damage. (K) Quantification of the $\gamma$-H2AX signals showed significant increase $(\approx 3$-fold) in the magnitude of damage for the +RGD:AuNP/+IR samples compared to the controls $(n=60$ /cohort). The values represent average $\pm \mathrm{SD}$. ****P $<0.0005$. 